Cycle Nucléaire. Assemblée Nationale-Senat, Paris, Juni 1998, 151 S., ISSN 1249-3872

Der Bericht kann bezogen werden vom Office Parlementaire d'Evaluation des Choix Scientifiques et Technologiques (OPECST), Assemblée Nationale, 233 Bd St Germain, F-75355 Paris Cedex 07 SP. Fax: ++ 33 (0) 140638808

》

\section{Xenotransplantation - Große Hoffnungen, hohe Hürden}

\author{
von Gerhard Samulat, Fraunhofer-Institut für \\ Systemtechnik und Innovationsforschung
}

\begin{abstract}
Jährlich erhalten allein in der Schweiz und in Deutschland rund 4000 Menschen ein Organ transplantiert. Nicht genug: Dreimal so viele Patienten stehen auf der Warteliste, denn menschliche Organe sind Mangelware. Einen Ausweg verspricht die Xenotransplantation, die Organverpflanzung vom Tier auf den Menschen. Der neuen Technik stellen sich indes noch hohe Hürden in den Weg.

Im Auftrag des Programms für Technikfolgen-Abschätzung des Schweizerischen Wissenschaftsrates hat das Fraunhofer-Institut für Systemtechnik und Innovationsforschung (ISI), Karlsruhe, erstmalig für den deutschsprachigen Raum, umfassend die Potentiale, aber auch Hemmnisse und Risiken der Xenotransplantation untersucht. Dabei wurden nicht nur die medizinischen Problemfelder beleuchtet, sondern insbesondere auch die gesellschaftlichen, ethischen und wirtschaftlichen Aspekte.
\end{abstract}

\section{Drei Barrieren}

Selbst ein Organ, welches innerhalb der gleichen Art - beispielsweise von einem Menschen auf einen anderen - übertragen wird, erkennt das Immunsystem des Empfängers als "fremd". Es greift das implantierte Organ an und stößt es nicht selten trotz starker medikamentöser Behandlung ab. Das gilt um so mehr für Organe, die von einem Tier auf den Menschen übertragen werden - man spricht bei diesen heftigen Reaktionen im Körper des Organempfängers von "hyperakuter Abstoßung". Mit Organen aus gentechnisch veränderten Schweinen hofft man nun, dieses Problem lösen zu können. Nach heutigem Stand der Forschung wären allerdings zur Zeit Versuche mit der Transplantation solcher (genetisch modifizierter) Schweineorgane auf den Menschen nicht vertretbar.

Noch weitgehend ungeklärt ist auch die Frage, ob Tierorgane überhaupt dauerhaft im Menschen überleben und dort lebenswichtige Funktionen übernehmen können. Ist doch der Hormon- und Stoffwechselhaushalt des Menschen anders als beim Schwein, das beispielsweise von Natur aus einen tieferen Cholesterinspiegel aufweist.

Außerdem ist grundsätzlich die Gefahr gegeben, mit den Xenotransplantaten bislang unbekannte Krankheiten auf den Menschen zu übertragen. Wissenschaftler befürchten beispielsweise, daß ähnliche Krankheiten wie AIDS oder die neue Variante der CreutzfeldJakob-Krankheit durch die Xenotransplantation auf den Menschen übertragen werden könnten. Die aktuelle internationale wissenschaftliche Diskussion zeigt jedenfalls, daß das Infektionsrisiko wesentlich größer sein könnte, als das noch vor wenigen Jahren vermutet wurde - mit möglichen Folgen und Kosten, die heute noch nicht überschaubar sind. Als besonders beunruhigend wird die Möglichkeit eingeschätzt, daß die Krankheitserreger nicht nur auf den Transplantatempfänger begrenzt bleiben, sondern auf Angehörige, auf Pflegepersonal und schließlich auf die ganze Gesellschaft übertragen werden könnten.

\section{Großes Konfliktpotential}

Nach Einschätzung des Fraunhofer-Instituts birgt die Xenotransplantation im Hinblick auf die ethischen, wirtschaftlichen, rechtlichen und gesellschaftlichen Aspekte ein erhebliches Konfliktpotential. Da aus medizinischen Gründen menschliche Organe bis auf weiteres den tierischen vorzuziehen sind, stellt sich beispielsweise die Frage nach den Auswahlkriterien: Wer bekommt das "bessere" menschliche Organ, wer das "schlechtere" tierische?

Ungeklärt ist auch die Frage, welche Kosten auf das Gesundheitswesen zukommen und wer diese übernehmen soll. Muß der Patient zahlen, besteht die Gefahr einer "Zwei-KlassenMedizin", weil sich nur vermögende Patienten die teure Behandlung leisten können. Zudem 
gilt es, das Interesse des einzelnen an einer lebensrettenden Xenotransplantation gegenüber dem Infektionsrisiko für die Gesellschaft abzuwägen.

Ferner müßten Spendertiere für die Xenotransplantation durch gentechnische Veränderungen soweit wie möglich auf das Immunsystem des Menschen "abgestimmt" und unter sterilen Bedingungen aufgezogen werden. $\mathrm{Ob}$ sich dies mit einer artgerechten Haltung und der in der Schweizerischen Verfassung verankerten Würde des Tieres vereinbaren läßt, ist zumindest fraglich.

\section{Vielversprechende Ansätze}

Anders sieht die Situation bei der Transplantation tierischer Zellen und Gewebe auf den Menschen aus. Beispielsweise wird in der Schweiz bereits untersucht, ob man Krebspatienten mit schweren, chronischen Schmerzen oder Patienten mit der - freilich seltenen - Nervenerkrankung amyotrophe Lateralsklerose durch Transplantation tierischer Zellen wirksam behandeln kann. Die Xenotransplantation von Zellen wird wohl sogar eher Praxisreife erlangen als diejenige von Organen. Zudem zielt sie auf Krankheiten, die viel weiter verbreitet sind als Organversagen. $\mathrm{Zu}$ nennen sind hier beispielsweise die Zuckerkrankheit (Diabetes mellitus), die Parkinson'sche Krankheit sowie verschiedene Krebserkrankungen, die zu chronische Schmerzen führen.

\section{Viele Türen stehen offen}

Im Hinblick auf die Transplantation von Organen stellen die Forscherinnen und Forscher aus dem Fraunhofer-Institut in Frage, ob es gerechtfertigt ist, eine derart komplexe Methode weiterzuentwickeln: die Erfolgsaussichten für eine breite Anwendung sind unsicher, auf die man sich ohnehin noch mindestens 15 bis 20 Jahre wird gedulden müssen. Aus Sicht der Wissenschaftler ist jedenfalls keineswegs gerechtfertigt, der Xenotransplantation einen besonderen Stellenwert zuzuweisen und sie als eine Art "Königsweg" darzustellen.

Das Augenmerk sollte vielmehr auf vorhandene Alternativen gelenkt werden. An erster Stelle sind Präventionsmaßnahmen zu nennen Bemühungen also, dafür zu sorgen, daß die
Organe und damit die Menschen von vornherein gesund bleiben. Außerdem sollte versucht werden, die Bereitschaft in der Bevölkerung zu stärken, sich als Organspender zur Verfügung zu stellen. Schließlich gibt es erste Ansätze zur Entwicklung künstlicher Organe.

Wegen der Konfliktträchtigkeit und wegen der großen gesellschaftlichen Tragweite der möglichen Folgen, regt das ISI an, die Xenotransplantation auf jeden Fall in einem breit angelegten öffentlichen Diskurs zu erörtern.

\section{Bibliographische Angaben}

Bärbel Hüsing, Eve-Marie Engels, Thomas Frick, Klaus Menrad, Thomas Reiss: TA 30/1998: Xenotransplantation. Bern: Schweizerischer Wissenschaftsrat

Schweizerischer Wissenschaftsrat: TA 30a/1998: Xenotransplantation - geprüft auf Herz und Nieren. Kurzfassung der TA-Studie "Xenotransplantation" auf deutsch, französisch und englisch. Bern: Schweizerischer Wissenschaftsrat.

Die Berichte können bei der TA-Geschäftsstelle bezogen werden (Fax: ++ 41-31-323 3659 oder über email: brigitta.walpen@swr.admin.ch).

\section{Kontakt}

Dr. Adrian Rüegsegger

Schweizerischer Wissenschaftsrat

TA-Geschäftsstelle

Tel.: ++ 41 (0) 31/3241458

E-mail: adrian.rueegsegger@swr.admin.ch

Dr. Bärbel Hüsing

Fraunhofer-Institut für Systemtechnik

und Innovationsforschung

Tel.: ++ 49 (0) 721/6809210

E-mail: sb@isi.fhg.de

Der Text wurde zuerst im Internet veröffentlicht: http://www.isi.fhg.de 